\title{
Enhancement of Equilibrium Shift in Dehydrogenation Reactions Using a Novel Membrane Reactor
}

\author{
Semi-Annual Report \\ September 1, 1997 - February 28, 1998
}

\author{
By \\ Shamsuddin Ilias \\ Franklin G. King
}

Work Performed Under Contract No.: DE-FG22-96PC96222

\author{
For \\ U.S. Department of Energy \\ Office of Fossil Energy \\ Federal Energy Technology Center \\ P.O. Box 880 \\ Morgantown, West Virginia 26507-0880
}

By

North Carolina A\&T State University

Department of Chemical Engineering

Greensboro, North Carolina 27411 


\section{Disclaimer}

This report was prepared as an account of work sponsored by an agency of the United States Government. Neither the United States Government nor any agency thereof, nor any of their employees, makes any warranty, express or implied, or assumes any legal liability or responsibility for the accuracy, completeness, or usefulness of any information, apparatus, product, or process disclosed, or represents that its use would not infringe privately owned rights. Reference herein to any specific commercial product, process, or service by trade

name, trademark, manufacturer, or otherwise does not necessarily constitute or imply its endorsement, recommendation, or favoring by the United States Government or any agency thereof. The views and opinions of authors expressed herein do not necessarily state or reflect those of the United States Government or any agency thereof. 


\title{
ENHANCEMENT OF EQUILIBRIUMSHIFT IN DEHYDROGENATION REACTIONS USING A NOVEL MEMBRANE REACTOR
}

\author{
Semi-Annual Technical Report \\ Reporting Period: 09/01/1997 through 02/28/1998 \\ Authors: Shamsuddin Ilias \\ Franklin G. King \\ Report Issue Date: 05/27/1998 \\ DE-FG22-96PC96222 - 03 \\ North Carolina A\&T State University \\ Department of Chemical Engineering \\ Greensboro, NC 27411 \\ Tel: (336) 334-7564 \\ Fax: (336) 334-7904
}




\begin{abstract}
Electroless deposition of palladium thin-films on a surface of microporous ceramic substrate has been used to develop a new class of perm-selective inorganic membrane. In our previous two reports, we presented mathematical models to describe transport of hydrogen the palladiumceramic composite membrane in single-stage permeation cell in cocurrent and countercurrent flow configurations. Analysis shows that the model equations have a singular point. In this report, a method is described how to avoid the singular point in order to solve the model equations numerically. To show the usefulness of the new method, a single-stage gas permeation for a three component system, without chemical reaction under cocurrent flow configuration has been used as an example.
\end{abstract}




\section{TABLE OF CONTENTS}

Title Page $\quad$ i

Disclaimer $\quad$ ii

$\begin{array}{ll}\text { Abstract } & \text { iii }\end{array}$

Table of Contents $\quad$ iv

Executive Summary $\quad$ v

$\begin{array}{ll}\text { Introduction } & 1\end{array}$

$\begin{array}{ll}\text { Results and Discussions } & 3\end{array}$

$\begin{array}{ll}\text { Conclusions } & 4\end{array}$

$\begin{array}{ll}\text { References } & 4\end{array}$ 


\section{EXECUTIVE SUMMARY}

In recent years, there has been increased interest in developing inorganic and composite membranes for in-situ separation of hydrogen to achieve equilibrium shift in catalytic membrane reactors. In this work, we are currently studying the use of palladium-ceramic membrane in membrane reactor for separation and recovery of hydrogen from a dehydrogenation reaction. In our previous reports we presented models to describe permeation of hydrogen through singlestage palladium-ceramic composite membrane cell. Analysis of the model equations show that there exists a singular point when permeate hydrogen mole fraction equals to reject hydrogen mole fraction. At this condition, the numerical solution fails. To overcome this, a new method is proposed in this report. An example of a cocurrent single-stage permeation cell with three component system, without chemical reaction has been used to demonstrate the usefulness of the proposed new method. 


\section{INTRODUCTION}

The overall objective of this project is to develop inorganic and composite membranes for in-situ separation of hydrogen and equilibrium shift in catalytic membrane reactors. The specific objectives of this research are to:

1. Design and fabrication of catalytic membrane reactor using thin film palladiumcomposite membrane for dehydrogenation of cyclohexane to benzene

2. Conduct dehydrogenation reaction experiments to study the equilibrium shifts and hydrogen permeation characteristics

3. Develop a theoretical foundation for equilibrium shifts and hydrogen transport in the membrane reactor

\section{MODEL DEVELOPMENTS}

In our previous reports we presented mathematical models to describe transport of hydrogen through palladium-ceramic composite membrane in a single-stage permeation cell using cocurrent and countercurrent flow configurations. Numerical results were compared with experimental permeation data. The results were also presented in recent US DOE sponsored symposium [1]. Analysis of the model equations show that there exist a singular point when the reject composition equals to the permeate composition. At this condition, the numerical scheme fails due to floating point error. A method is proposed to overcome this numerical instability. A cocurrent flow configuration is used as an example to show the usefulness of the new method.

\section{Single-Stage Gas Permeation without Reaction}

The mathematical formulation for single-stage gas permeation process is based on following assumptions: (a) there are two permeable components $a$ and $b, a$ is more permeable than $b$, and one non-permeable component $i$ in the system; (b) the permeability of each gas component is the same as that of the pure gas, and is independent of pressure; (c) negligible gas phase concentration gradients exists in the permeation direction; (d) negligible pressure drop in the feed and permeate gas streams; (e) negligible diffusion along the axial flow path; (f) plug flow and ideal gas are assumed for both feed and permeate streams and (g) permeation obeys modified Fick's law. The flow stream and its compositions for co-current flow are shown schematically in Fig. 1.

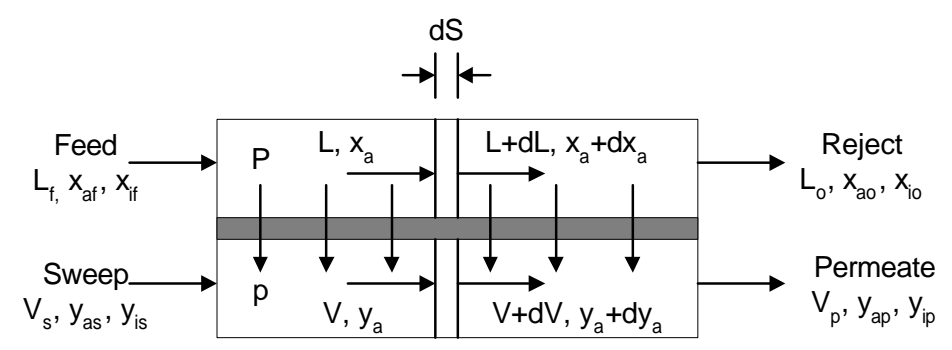

Figure 1: Schematic of cocurrent permeation cell 
Using the permeation rate equations and a material balance over the differential area $d S$, the following differential equations can be obtained:

Permeation rate equations of $a$ and $b$ :

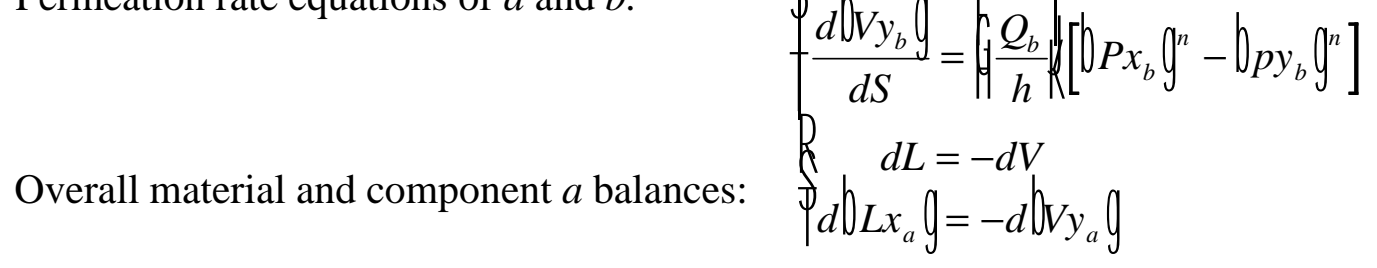

The overall mass and component balances between the inlet and at any arbitrary point along the membrane are given as:

$$
\begin{array}{ll}
L_{f}+V_{s}=L+V & L x_{i}=L_{f} x_{i f}=\text { constant } \\
L_{f} x_{a f}+V_{s} y_{a s}=L x_{a}+V y_{a} & V y_{i}=V_{s} y_{i s}=\text { constant }
\end{array}
$$

Combining Eqns. (1) and (2), and defining selectivity, $\alpha=Q_{a} / Q_{b}$, pressure ratio, $\gamma=p / P$, flow rate ratio, $F_{f}=V_{s} / L_{f}$, and dimensionless membrane area, $R=Q_{a} P^{n} S / h L_{f}$, one can obtain the following differential equations::

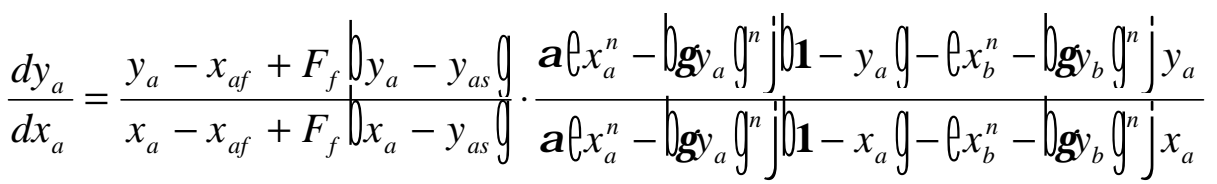

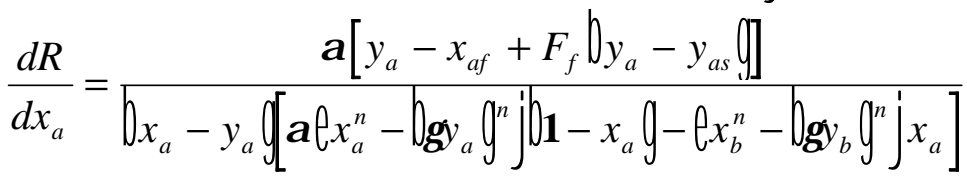

Eqns. (3) and (4) were solved by the Runge-Kutta-Gill method as an initial value problem with the boundary conditions:

$$
\text { At } R=\mathbf{0}: \begin{array}{lll}
\mathbf{Q}=L_{f} ; & x_{a}=x_{a f} ; & x_{i}=x_{i f} \\
=V_{s} ; & y_{a}=y_{a s} ; & y_{i}=y_{i s}
\end{array}
$$

For the special case of no sweep gas on the permeate side, $y_{a s}$ can not be specified and must be determined by Eqns. (3) and (4) subject to boundary conditions, Eqn.(5), where $V_{s}=\mathbf{0}$ and $y_{i s}=\mathbf{0}$. At the feed inlet, $y_{\text {as }}$ can be expressed as:

$$
\frac{y_{a s}}{1-y_{a s}}=\alpha \frac{x_{a f}^{n}-\bigotimes_{a s} \mathbf{9}}{x_{b f}^{n}-\bigotimes_{b s} \mathbf{9}}
$$


It is noted that Eqn.(3) appears to be indeterminate at the feed inlet for no sweep gas. Using L'Hospital's rule, the differential equation at the feed inlet can be obtained as:

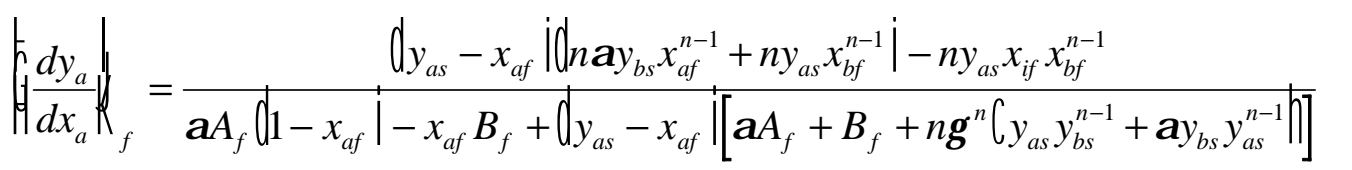

where $A_{f}=x_{a f}^{n}-\bigotimes_{a s} C_{\text {and }} B_{f}=x_{b f}^{n}-\boldsymbol{\bigotimes}_{b s} \mathbf{Q}$

\section{MODEL ANALYSIS FROM THE VIEWPOINT OF STABILITY}

Equations (3) and (4) appear to be indeterminate at the singular point:

$$
x_{a}=y_{a}=\frac{x_{a f}+y_{a s} F_{f}}{\mathbf{1}+F_{f}}
$$

Numerical scheme becomes unstable at this point. Pan and Habgood [2] also identified the same singular point. Their analysis, however was away from singular point. One can avoid this singular point by an appropriate selection of the operating variables. Following example will illustrate this phenomenon.

\section{Example:}

\begin{tabular}{|c|c|c|}
\hline Feed Gas Condition & Sweep Gas Condition & Parameters \\
\hline $\mathrm{x}_{\mathrm{af}}=\mathbf{0 . 7 1}$ & $\mathrm{y}_{\mathrm{as}}=0.26$ & $\mathrm{n}=1.0$ \\
$\mathrm{x}_{\mathrm{if}}=0.08$ & $\mathrm{y}_{\mathrm{is}}=0.30$ & $\alpha=650$ \\
$\mathrm{~L}_{\mathrm{f}}=1.0$ & $\mathrm{~V}_{\mathrm{s}}=2.0$ & $\gamma=0.1$ \\
\hline
\end{tabular}

Figure 2 shows plots of reject $D_{a}$ ! and permeate $D_{a}$ ! mole fractions as a function of dimensionless membrane area, $R$. The plots intersect at the singular point, where $x_{a}=y_{a}=0.41$. This singular point can be avoided if composition of the more permeable component in feed gas and sweep gas is at either side of this point. So, there are two regions for which singular point will not arise: $y_{a s}>x_{a f}$, then the composition of the more permeable component in sweep gas is greater than that in the feed inlet; and 0 g $y_{a p}<x_{a o}$, then the composition of more permeable component in permeate is less than that in the retentate.

The following procedure can be used to obtain numerical results for the whole range of stage cuts. Generally, an increment, like $10^{-n}$ with an integer exponent is used. As the singular point is approached, the numerical method will fail. In that case if one uses a factor 1.1, 1.3, 1.7 etc. with $10^{-n}$, the instability can be avoided. The data reveals that there are some discrepancies around the singular point. This is shown graphically in Figure 3 . The inset of the figure shows the behavior of the function near the singular point. 


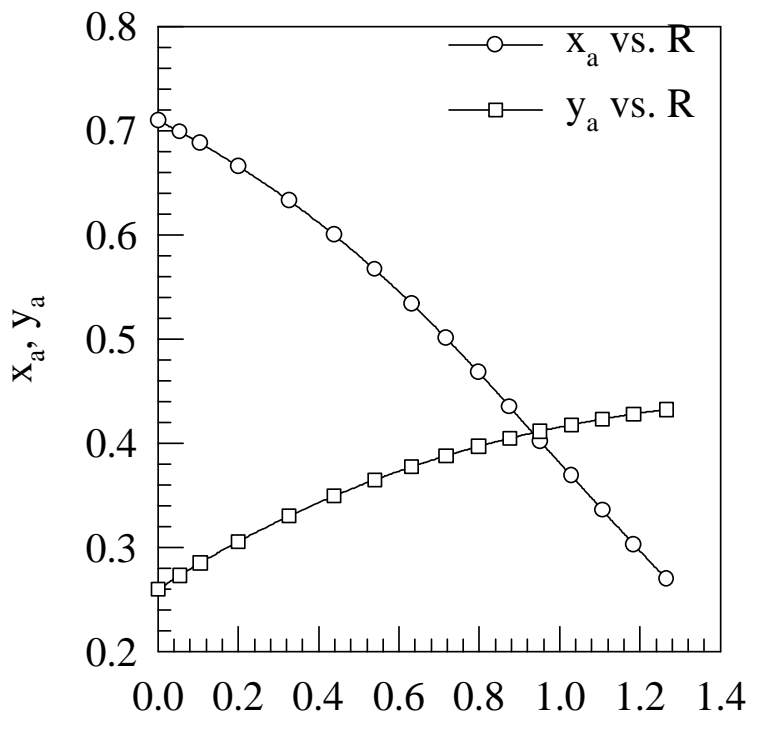

Dimensionless Membrane Area, $\mathrm{R}$

Figure 2: Reject and permeate mole fractions as function of dimensionless membrane area.

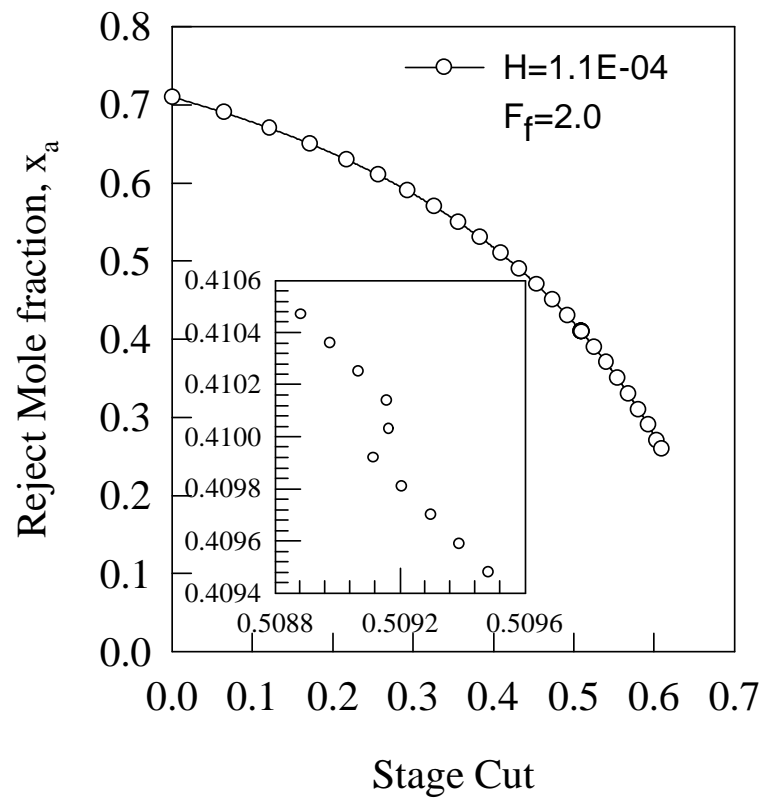

Figure 3: Efect of stage cut on reject mole fraction for cocurrent flow (singularity is shown in the insert figure).

\section{CONCLUSIONS}

From physical point of view, the solution of eqns. (1) and (2) should be continuous at $x_{a}=y_{a}$. But these two equations are not defined at $x_{a}=y_{a}$. So, to solve these equations numerically, care should be taken to avoid this singular point. At this point floating point error may or may not occur. Floating point error does not occur if the singular point contains repeated decimal point. In other cases floating point error would occur and this error can be avoided by appropriate selection of increment.

\section{REFERENCES}

1. Mondal, A.M., King, F.G., and Ilias, S., Proc. $6^{\text {th }}$ Annual HBCUs/OMIs Program Energy Research and Development Technology Transfer Symposium, Ocean City, MD., April 2729, 1998.

2. Pan, C. Y., and Habgood, H. W., Ind. Eng. Chem. Fund., 13, 323(1974). 